\title{
Preface to the CLEI 2019 Special Issue
}

\author{
Vladimir Villarreal \\ Universidad Tecnológica de Panamá \\ vladimir.villarreal@utp.ac.pa \\ Gabriela Marín-Raventós \\ Universidad de Costa Rica \\ gabriela.marin@ucr.ac.cr \\ Héctor Cancela \\ Universidad de la República \\ cancela@fing.edu.uy
}

This special issue of the CLEI Electronic Journal (CLEIej) consists of extended and revised versions of Selected Papers presented at the 44rd Latin American Conference in Informatics (CLEI 2019), held in Panama City, Panama, September $30^{\text {th }}$ to October $4^{\text {th }}, 2019$.

CLEI 2019 received 234 full paper submissions for its nine different Symposia

- Latin American Symposium on Software Engineering

* Latin American Symposium on Operations Research and Artificial Intelligence

* Latin American Symposium on Computing and Society

* Latin American Symposium on Data Management Systems

- Latin American Symposium on Business Processes, Architectures and Enterprise Systems

- Latin American Symposium on Computer Graphics, Virtual Reality, and Image Processing

- Latin American Symposium on Infrastructure, Hardware and Software

- Latin American Symposium on Theory of Computation

- XXVII Iberoamerican Congress on Higher Education in Computing

After a thorough review process involving 350 program committee members, and an average of 3.18 reviews per paper, 84 submissions were accepted, for a $35.9 \%$ acceptance rate.

This special issue contains papers selected among those presented in the CLEI 2019 conference, considering its quality and contribution. The authors of 11 selected papers were invited to extend and include novel contributions. Extended versions went through another round of reviews. The accepted articles are introduced.

In the first article, "Tendencies in Multi-Agent Systems: A Systematic Literature Review", Mariana Falco and Gabriela Robiolo present a systematic literature review on Multi-Agent Systems (MAS), following Kitchenham and Wholin guidelines. It is very useful to understand the progress of MAS from 2009 to the present days.

In the second article, "A Real-Time Entity Monitoring based on States and Scenarios", Mario Jose Divan and Maria Laura Sanchez Reynoso present PAbMM, a real-time processing architecture specialized in measurement projects associated with a measurement and an evaluation framework. 
The third article, "Model-driven Support for Business Process Families with the Common Variability Language (CVL)" by Daniel Calegari, Andrea Delgado and Leonel Peña, explores the use of CVL together with the Business Process Model and Notation (BPMN 2.0) for modeling business process families, and the use of ModelDriven Engineering (MDE) techniques for the automatic generation of process variants using a graphical tool.

The fourth article, "Semantic Segmentation of 3D Medical Images with 3D Convolutional Neural Networks" by Alejandra Marquez Herrera, Alex Cuadros-Vargas and Helio Pedrini, describes research done to improve the task of binary semantic segmentation of volumetric medical images acquired by Magnetic Resonance Imaging (MRI). It uses a pre-existing Three-Dimensional Convolutional Neural Network (3D CNN) architecture and a loss function for training this 3D CNN.

In the last article, "A research model in didactics of programming" by Sylvia da Rosa and Federico Gomez, a research model in didactics of programming is presented. It is elaborated within the theoretical framework of the epistemological theory of Piaget. The paper describes a research model on students' construction of knowledge about computer programs.

We would like to thank all the members of the community who were involved in this special issue, and particularly the reviewers for their anonymous contribution to the selection process which ensured the quality of the selection. 